\title{
Direction et fonctionnement des établissements
}

Le cas de la Thuringe

Horst Weishaupt, Monika Plath et Ilona Böttcher

Traducteur : Dominique Gélin

\section{OpenEdition}

\section{Journals}

Édition électronique

URL : http://journals.openedition.org/ries/4195

DOI : 10.4000/ries.4195

ISSN : 2261-4265

\section{Éditeur}

Centre international d'études pédagogiques

\section{Édition imprimée}

Date de publication : 1 décembre 1994

Pagination : 81-94

ISSN : 1254-4590

\section{Référence électronique}

Horst Weishaupt, Monika Plath et Ilona Böttcher, « Direction et fonctionnement des établissements », Revue internationale d'éducation de Sèvres [En ligne], 04 | 1994, mis en ligne le 17 avril 2015, consulté le 06 janvier 2020. URL : http://journals.openedition.org/ries/4195 ; DOI : 10.4000/ries.4195

Ce document a été généré automatiquement le 6 janvier 2020

(c) Tous droits réservés 


\title{
Direction et fonctionnement des établissements
}

\author{
Le cas de la Thuringe \\ Horst Weishaupt, Monika Plath et llona Böttcher \\ Traduction : Dominique Gélin
}

1 La Thuringe a complètement restructuré son système éducatif. Dans l'ancienne République démocratique allemande (RDA), il consistait en dix années d'école polytechnique d'enseignement général obligatoire (POS), suivies de deux ans de second cycle élargi (EOS). Le second cycle élargi qui menait à l'Abitur concernait moins de $15 \%$ seulement d'une classe d'âge. Ce système d'école unique a disparu après la dissolution de l'État centralisé de RDA lui-même. On a adopté à la place la structure fédérative de la RFA, avec un système éducatif qui est un élément essentiel des compétences des Länder.

2 La Thuringe, un des cinq nouveaux Länder, a remplacé à la rentrée scolaire 1991 le système unique par une structure articulée, avec des parcours différents selon les niveaux : après les quatre années d'école élémentaire communes à tous, la scolarité se poursuit soit en Regelschule, soit au Gymnasium. La Regelschule, où l'on peut obtenir les diplômes de fin d'études de Hauptschule et de Realschule, est considérée comme le «noyau central » de la nouvelle structure. Ceci, non seulement parce qu'environ deux tiers des élèves du premier cycle secondaire la fréquentent, mais surtout parce que la Thuringe réunit là dans une même structure les élèves qui, dans les anciens Länder, fréquentent des établissements différents, les Haupt- et Realschule. Ce faisant, la Thuringe - tout comme la Saxe et le Land de Saxe-Anhalt - franchit un pas dans l'organisation du premier cycle secondaire, qui doit amener à la fin de la confrontation stérile entre le système articulé à trois voies et la Gesamtschule ${ }^{1}$. 


\section{Les effets de la réunification}

\section{Modification dans la structure de la gestion des établissements}

3 En Allemagne, les systèmes éducatifs sont traditionnellement une organisation étatique et bureaucratique. Toutefois "la tendance à la domination de l'État sur l'école, traditionnelle en Allemagne, avait atteint son point culminant en RDA dans le monopole de l'État, illimité et exercé de façon centralisée.» (Jenkner, 1989, p. 47). «Toutes les questions essentielles de fond et les questions matérielles concernant l'organisation de l'école étaient réglées de façon uniforme, dans l'ensemble et jusqu'au moindre détail » par la direction du parti et le ministère de l'éducation au niveau central de l'État (Jenkner, 1989, p.48). Les départements éducatifs des conseils de district et des cantons étaient responsables de la mise en œuvre des décisions du parti et du gouvernement. Il y avait certes dans les écoles des droits de proposition, de consultation et de participation pour les enseignants, les parents et les associations qui travaillaient en partenariat avec les établissements, mais le chef d'établissement était le seul habilité à prendre des décisions et devait rendre des comptes aux échelons supérieurs. Le travail en équipe au sein du collectif enseignant, concernant les activités pédagogiques et extrascolaires, revêtait une grande importance pour chaque enseignant. Les lourdeurs formelles pouvaient ainsi, dans une certaine mesure, être relativisées par des processus de communication et de coopération au sein de groupes d'enseignants.

4 Avec la nouvelle structure scolaire, on a non seulement rompu avec le principe d'unicité des compétences, en introduisant le "double subventionnement ", c'est-àdire la répartition des compétences pour chaque école en affaires « intérieures », prises en charge par le ministère de l'éducation, et "extérieures", dépendantes des subventions du district. Mais, ce qui est encore bien plus important pour le travail dans les établissements, on a élargi les droits de coopération des enseignants, des parents et des élèves. Les enseignants jouissent, par ailleurs, d'un bien plus grande autonomie dans l'organisation de leurs cours. Parallèlement l'établissement s'est vu attribuer, en tant qu'unité pédagogique, des espaces de liberté pour la définition de son propre profil. La position du chef d'établissement vis-à-vis du corps professoral a aussi été modifiée : il n'est plus que primus inter pares. Mais un rôle d'autant plus central lui revient pour faire évoluer l'établissement, en tant qu'initiateur de multiples processus pédagogiques.

Une difficulté de cette nouvelle loi scolaire tient à l'accent mis sur l'individualité de l'enseignant, qui peut entraîner l'isolement des enseignants concentrés principalement sur leurs propres cours. Cette observation a conduit la sociologie de l'école à revoir sa vision de l'école :

Même si l'école ne manque pas d'éléments bureaucratiques - qui assurent dans une certaine mesure les fonctions de répartition des ressources et de liaison avec l'extérieur, indispensables à sa survie-, cette institution est [...] avant tout caractérisée par une structure quasi féodale de domaines d'actions et de dispositions «sans liens réels», ainsi que par une organisation «cellulaire» du travail. (Baumert ; Leschinsky, 1986, p. 248)

6 Il existe en effet fort peu de contacts institutionnalisés entre les enseignants, si bien que l'ensemble a du mal à trouver une cohérence forte. La mission de la communauté scolaire - et là le rôle du chef d'établissement revêt une importance particulière - est 
donc d'aller à l'encontre d'un "combat individualiste", qui n'est plus que supervisé bureaucratiquement, en prenant de nombreuses mesures favorisant l'organisation et l'évolution de l'école.

Dans ce contexte on pourrait considérer que la modification essentielle dans le système éducatif de Thuringe est le passage d'un système unique, centralisé, à un système pluraliste, avec une relative autonomie de chaque établissement. L'objectif de la gestion et de l'évolution de chaque établissement est d'empêcher l'isolement des enseignants et de convaincre le corps professoral et la communauté scolaire de participer à la définition du profil de l'établissement.

\section{Problèmes spécifiques au développement de la Regelschule}

8 Alors que l'école élémentaire et le Gymnasium peuvent trouver des modèles en Allemagne de l'Ouest pour leur travail pédagogique, les chefs d'établissement de Regelschule sont contraints dans une large mesure de donner un profil pédagogique spécifique à cette structure de conception nouvelle.

$9 \quad$ Les principaux aspects en sont les suivants.

\section{La modification des données structurelles}

10 Il est possible de passer de la Regelschule au Gymnasium en fin de cinquième et de sixième année d'école. Il s'agit là d'une correction possible des orientations de fin d'école élémentaire pour les "élèves qui se révèlent tardivement ». Mais de plus en plus d'élèves sont aussi réorientés vers la Regelschule, en provenance du Gymnasium, parce qu'ils ne sont pas au niveau des exigences. L'intégration de ces élèves représente un défi de plus en plus important pour la Regelschule.

11 En outre, des corrections de carte scolaire s'avèrent nécessaires a posteriori. Au lieu des 457 Regelschulen de l'année scolaire 1991-1992, il n'y en avait plus que 402 en 1993-1994. D'autres sites d'implantation sont menacés. D'après les prescriptions du ministère, les Regelschulen doivent avoir au moins trois classes parallèles par niveau ; l'établissement ne peut qu'exceptionnellement avoir deux classes. Il règne de ce fait dans de nombreux établissements une certaine incertitude quant aux futures conditions de travail, soit parce qu'un établissement risque la fermeture, soit parce qu'il devra accueillir les élèves et les enseignants d'un établissement qui fermera.

\section{La définition du profil à l'intérieur de chaque établissement}

Parallèlement à la réorganisation des structures de ces établissements, on a expérimenté de nouveaux contenus, du moins dans certaines matières: économie et environnement en Europe, économie et technique, économie et droit, sciences sociales, religion et éthique. Dans un premier temps, il n'y a eu que des « indications provisoires de programmes " pour aider les enseignants à structurer leurs cours; depuis l'année scolaire 1993-1994, on fonctionne sur des «instructions officielles provisoires». À la base des nouvelles instructions: de nouveaux horaires, au sein desquels le poids accordé à certaines matières a été revu. Pour une meilleure connaissance du futur monde du travail, les élèves font des stages dans des entreprises qu'ils ont choisies. 
13 On s'est posé des questions, en même temps qu'on redéfinissait les contenus, sur les procédures et les démarches. Dans « l'école unique ", les enseignants avaient l'habitude de classes très hétérogènes. La "tête de classe ", qui faisait souvent fonctionner le cours en POS, manque très nettement dans les classes de Regelschule. Un des nouveaux enjeux pour les enseignants est donc un enseignement adapté aux besoins de groupes d'élèves dont les niveaux sont relativement homogènes. Le problème est particulièrement aigu dans les groupes d'élèves qui relèvent de la Hauptschule, en raison de leur faible niveau.

\section{Activités extrascolaires et vie de l'école}

14 Comme le montrent les résultats d'une enquête que nous avons menée dans les établissements de Thuringe, plus particulièrement les parents des élèves scolarisés en Regelschule attendent de l'école qu'elle joue un rôle éducatif et souhaitent qu'on leur propose des activités extrascolaires. Les activités extrascolaires, sur le temps libre, n'étaient pas une des moindres caractéristiques des POS.

15 Actuellement, les enseignants et les chefs d'établissement essaient de répondre au mieux aux attentes des parents avec des moyens matériels et humains nettement moins importants. En même temps, les Regelschulen doivent affirmer leur spécificité dans la concurrence avec le Gymnasium et chercher des moyens pour augmenter leur " cote " auprès des parents. Dans ce but, on propose, comme partie intégrante de la vie de l'établissement, différentes manifestations et temps forts qui viennent compléter l'enseignement.

\section{Mutation de l'école et mutation de la société}

16 Les conditions particulières de la mise en place des Regelschulen, telles que nous venons de les décrire, doivent être considérées en liaison étroite avec le processus de mutation de l'ensemble de la société dans les nouveaux Länder. Tous les domaines sociaux subissent de profondes transformations qui nécessitent de la part de tous les acteurs un changement de mentalité et des capacités d'adaptation. Il en résulte actuellement des dysfonctionnements nombreux et complexes car le changement des mentalités et l'évolution de la prise de conscience ne sont pas concomitants des changements institutionnels et structurels. À cet égard, les processus d'organisation scolaire en Thuringe diffèrent fondamentalement de l'évolution des établissements dans les anciens Länder.

Il faut ajouter à tout cela le fait que les personnes concernées n'ont eu à aucun moment l'occasion de participer à la refonte des structures. Enseignants et parents ont la plupart du temps subi les mutations occasionnées par le « changement politique » et se sont, dans un premier temps, montrés très sceptiques à leur égard ${ }^{2}$. Les enseignants ont du mal à se défaire de leurs habitudes ou retombent inconsciemment dans des schémas comportementaux qui leur sont familiers, mais qui sont « dépassés ». Il faut s'attendre à de tels hiatus entre les nouvelles structures et d'" anciens " habitus dans l'action des chefs d'établissement pour organiser et définir le profil de la Regelschule.

Dans l'optique de ce changement des structures scolaires, il est particulièrement significatif de savoir que presque tous les chefs d'établissement ont été installés dans cette fonction lors de la période de changement politique. Sur les $236 \mathrm{chefs}$ 
d'établissement de Thuringe que nous avons interrogés, $4 \%$ seulement occupaient ce poste déjà avant 1990, un tiers a pris ses fonctions en 1990, $59 \%$ en 1991 et $4 \%$ en 1992-1993. Si on différencie selon les types d'établissements, on remarque que la moitié des chefs d'établissement de Regelschule et Gymnasium ont déjà été nommés en 1990 alors que les directeurs d'école primaire n'ont pris leurs nouvelles fonctions qu'en 1991, avec l'introduction du système articulé.

Il faut souligner le pourcentage, $53 \%$, de femmes chefs d'établissement. Elles sont néanmoins sous-représentées si on se réfère à la proportion de femmes enseignantes, qui s'élève à environ $75 \%$. D'après les résultats de notre enquête auprès des chefs d'établissement, on constate des différences importantes selon les types d'écoles : il y a $80 \%$ de femmes directrices d'école primaire, $31 \%$ de femmes qui dirigent une Regelschule et seulement $7 \%$ à la tête d'un Gymnasium.

\section{Les nouveaux chefs d'établissement}

\section{Importance du chef d'établissement pour le fonctionnement de l'établissement}

20 Les compétences des chefs d'établissement en matière de direction et de conseil sont absolument décisives pour l'évolution d'un établissement, afin que tous les éléments nécessaires à un bon travail pédagogique puissent être rassemblés et mis en cohérence. On distingue six grandes orientations et capacités qui sont des critères importants pour une bonne direction et, par voie de conséquence, pour qu'un établissement devienne un « bon » établissement :

- la capacité à défendre les objectifs d'un établissement afin d'obtenir un consensus du corps professoral pour leur mise en œuvre, ainsi que la capacité à veiller au respect des décisions et des orientations prises ;

- la capacité à mettre au centre de la vie scolaire et du travail des enseignants la réalisation du projet pédagogique de l'établissement ;

- cela suppose que le chef d'établissement demeure vraiment inscrit dans le processus de communication et de coopération du corps professoral et « dégage » du temps pour cela ;

- d'où une quatrième capacité, la compétence organisationnelle ;

- et, au-delà, des capacités et dispositions pour conseiller les collègues, leur ouvrir des espaces de liberté permettant des initiatives pédagogiques, tout en protégeant autant que possible ces espaces de liberté des tentatives réductrices extérieures ;

- enfin, dispositions et capacité pour ancrer l'établissement dans son environnement communal, pour l'ouvrir sur l'extérieur, pour le mettre en relation avec d'autres établissements et pour favoriser la collaboration avec les parents, sans pour autant transformer l'établissement en simple lieu d'exécution des vœux et des opinions des parents. (Klafki, 1991, p. 38)

\section{Les Regelschulen choisies pour l'enquête}

21 À partir de ces critères, trois exemples vont nous permettre d'observer plus précisément comment des chefs d'établissement réussissent à accomplir cette mission de direction. Pour cela on tiendra compte de la combinaison de plusieurs facteurs : les expériences de ces chefs d'établissement dans leur ancienne activité professionnelle, 
leur degré de socialisation en tant qu'enseignant et leur façon de répondre aux nouvelles exigences. Trois critères ont présidé au choix des Regelschulen.

enquête n'ont pris leurs fonctions qu'en 1990 ou 1991, ils n'ont donc qu'une expérience réduite de cette tâche. La restructuration complète du système éducatif les a immédiatement placés en face de vastes problèmes de fonctionnement. Le fait que la création de la Regelschule n'ait pas été le résultat de débats sur la politique scolaire ayant associé des enseignants, mais de discussions politiques menées "par-dessus la tête des enseignants", a sérieusement compliqué la tâche, car de nombreux enseignants ont du mal, dans ces conditions, à accepter les nouvelles exigences de contenu et de méthode.

L'action des chefs d'établissement est analysée en fonction des six dimensions évoquées précédemment. 


\section{Défense et mise en œuvre des objectifs} pratique des objectifs pédagogiques. Le corps professoral, issu de trois établissements, manque nettement d'homogénéité, le travail en commun est encore peu développé chez les enseignants. Beaucoup d'entre eux occupaient un poste à responsabilité lors de la période antérieure (RDA); ils ont dû abandonner ces fonctions au moment du " changement politique », mais n'ont pas été contraints de quitter l'enseignement. Les entretiens dans cet établissement ont révélé un manque d'intégration et de coopération du corps professoral qui se traduit principalement par un manque de cohérence dans les réponses des enseignants face aux problèmes de discipline. 
33 Par contre, il est impressionnant de voir comment «la » chef d'établissement B parvient à aligner l'action pédagogique au quotidien sur les objectifs pédagogiques, au moyen de décisions prises en assemblées générales pour concertation entre enseignants. Un exemple : le niveau globalement faible des élèves de Hauptschule pose problème aux enseignants car ils avaient jusqu'ici seulement l'expérience de l'enseignement dans des classes hétérogènes. Ces difficultés ont poussé le chef d'établissement à mettre en place un programme pédagogique spécial, destiné à ces élèves qui "nécessitent une attention toute particulière », afin de prendre mieux en compte leurs acquis antérieurs et de leur donner la possibilité d'éprouver un sentiment de réussite à l'école. Au-delà de cet exemple précis, les enseignants s'efforcent d'organiser leurs cours en tenant compte des intérêts des élèves.

Dans cet établissement, la centration sur l'élève se concrétise aussi par la prise en charge des élèves réorientés, en provenance du Gymnasium. Ces élèves sont non seulement déstabilisés par leur échec au Gymnasium, mais ils ont de surcroît des lacunes à combler entre la huitième et la dixième classe de la Regelschule, en raison des horaires-matières différents au Gymnasium et en Regelschule. La direction a insisté pour développer également des actions en collaboration avec les parents, qui permettent une meilleure intégration sociale des élèves dans la nouvelle école et une amélioration stable des résultats.

Cet établissement fait porter tous ses efforts sur l'orientation afin qu'elle s'inscrive dans la durée et qu'elle responsabilise les élèves. À la fin de la sixième classe, les élèves doivent être répartis entre Hauptschule et Realschule ${ }^{3}$. Le chef d'établissement est partie prenante du processus d'orientation; dans cette perspective, elle assiste à une matinée de cours dans chaque classe - sur la base des propositions du professeur principal - et donne ensuite son avis pour la prise de décision définitive ${ }^{4}$.

Le chef d'établissement $C$ souligne - en plus des réunions formelles - l'importance des concertations informelles entre enseignants pour la mise en œuvre du projet pédagogique. Ce type de concertation s'impose étant donné le nombre réduit d'enseignants dans cet établissement, d'autant plus que la nécessité d'une concertation institutionnalisée - par exemple entre les enseignants d'une même matière - est moins forte que dans un établissement de plus grande taille. Mais cela entraîne une absence de décisions engageant tout le corps professoral sur des principes d'actions pédagogiques communs et un défaut de processus de clarification et de régulation nécessaires à la cohésion de l'ensemble. Dans un établissement où, après le " changement », le corps professoral a été certes réduit mais est néanmoins demeuré stable, le changement de cap n'est pas concevable sans des discussions et des initiatives $\mathrm{du}$ chef d'établissement. Or le chef d'établissement n'a pas réussi à faire passer ce message. Dans les réponses des personnes interrogées, on perçoit encore une sorte d'« état d'esprit latent de POS » : ainsi, les enseignants de cet établissement éprouvent encore beaucoup de difficultés à s'adapter aux élèves de Hauptschule dont les résultats sont homogènes vers le bas. La décision de maintenir tels quels les groupes classe ressort plutôt d'une façon de s'accrocher aux méthodes pédagogiques qui étaient en usage dans les POS que d'une décision consciente d'enseignement différencié (dans cet établissement on ne pratique pas la différenciation dans les matières principales). Bon nombre d'enseignants de cet établissement, encore imprégnés du « rôle directif de l'enseignant ", ont beaucoup de mal à prendre en compte la parole des élèves, leurs revendications, et à en tenir compte dans l'organisation de leurs cours. On ne peut 
s'empêcher d'avoir l'impression que la cohésion du corps professoral - chef d'établissement compris - qui a résisté au "changement», a jusqu'ici largement entravé tout travail de réflexion critique sur la pratique pédagogique du temps de la RDA.

\section{Inscription dans le processus de communication et de concertation des enseignants}

En 1993, nous avons demandé à environ 1300 enseignants de Thuringe, entre autres choses, s'ils étaient satisfaits de leur travail actuel à l'école. Le résultat a été que les enseignants étaient surtout satisfaits de leurs supérieurs hiérarchiques (chefs d'établissement), plus que de leurs collègues. Il faut interpréter ce résultat de la façon suivante: les chefs d'établissement sont reconnus comme compétents, ils ont un bon contact avec les enseignants et les enseignants trouvent une oreille attentive à leurs préoccupations. Les chefs d'établissement des Regelschulen sélectionnées pour l'enquête sont, eux aussi, perçus positivement par leurs enseignants. Si l'on compare les trois établissements, l'appréciation portée sur la direction par les enseignants est de plus en plus positive quand on va de A vers $C$. L'appréciation relativement négative portée par les enseignants de l'établissement A sur leur direction s'explique par les difficultés organisationnelles décrites plus haut. S'ajoute à cela le fait que l'établissement n'a pas de salle des professeurs. Les enseignants n'ont que trois petites salles à leur disposition, ce qui limite très nettement les occasions de communication collégiale et le chef d'établissement n'a quasiment pas de possibilités de s'y intégrer.

Le style de communication du chef d'établissement B avec les enseignants est caractérisé par un équilibre entre intégration et distance. Par contre le chef d'établissement $\mathrm{C}$ a tissé au fil des ans des liens avec de nombreux collègues, elle est de ce fait intégrée et appréciée de ses enseignants. Mais ces liens collégiaux font qu'elle manque d'assurance pour faire passer ses propres objectifs de gestion de l'établissement.

\section{Compétence organisationnelle}

39 Nous avons soumis aux 236 chefs d'établissement de Thuringe interrogés, une liste de problèmes concernant les conditions propres à l'organisation des cours, la didactique et la discipline ou l'éducation ainsi que les conditions matérielles. Les domaines où les problèmes sont considérés comme essentiels (et là il n'y a aucune différence entre les types d'établissements) sont les suivants: moyens financiers insuffisants, état de délabrement du bâtiment, état des salles de classe, équipement mobilier.

D'après eux, tous les problèmes concernant les élèves et l'enseignement sont, en comparaison, de moindre importance. Ce résultat s'explique par le mauvais état de la plupart des bâtiments scolaires en Thuringe, tant à l'extérieur qu'à l'intérieur. Les établissements sélectionnés pour l'enquête ont, eux aussi, fortement besoin d'être rénovés. Leur équipement est nettement insuffisant, compte tenu des nouveaux programmes et de l'introduction de nouvelles matières.

41 Les trois chefs d'établissement se sont beaucoup investis dans la rénovation progressive des bâtiments et de l'environnement immédiat, ainsi que dans l'amélioration de l'équipement et du matériel pédagogique. Ils ont tous les trois des contacts réguliers et personnels avec les administrations du district et des communes, responsables des 
constructions et de l'équipement. Dans l'établissement B, l'Association de soutien, créée en grande partie à l'initiative du chef d'établissement, est un partenaire important pour obtenir des moyens supplémentaires. Avec son aide, on a pu installer une nouvelle salle informatique et deux salles de travail supplémentaires. Ce qui permet à l'établissement de faire sa propre publicité.

Le chef d'établissement A doit faire preuve de talents organisationnels particuliers pour faire en sorte que les emplois du temps coïncident avec les horaires des cars dont dépendent les élèves.

\section{Conseiller et assurer des espaces de liberté}

Les propositions d'activités extrascolaires sont une part importante du programme des trois établissements. Les enseignants et les élèves peuvent y satisfaire leurs intérêts spécifiques en dehors de tout programme et de toute matière. Le chef d'établissement $\mathrm{A}$ reconnaît que cette offre d'ateliers est une voie possible pour définir le profil de l'établissement, mais il rencontre des difficultés importantes pour les ouvrir à tous les élèves, en raison des contraintes horaires des transports scolaires. Dans l'établissement C, la mise en place des ateliers dépend d'heures mises à la disposition des enseignants, mais ces heures sont en partie utilisées pour des remplacements. Les ateliers risquent donc d'être considérés comme un supplément dont on peut se passer et non comme partie intégrante de la vie de l'établissement. Dans l'établissement B, il y a également un large éventail d'activités extrascolaires, les élèves étant associés aussi bien à l'organisation qu'à la définition des contenus.

Les trois chefs d'établissement soutiennent l'intérêt de la majorité de leurs enseignants pour des séminaires de formation continue qui permettent d'acquérir de nouvelles qualifications et compétences. Les enseignants de sciences sociales et de russe, langue qui n'est presque plus choisie par les élèves, ont un besoin urgent de recyclage. Les directions sont favorables à tous les stages qui traitent de pédagogie, de droit de l'école et de didactique des disciplines. Le chef d'établissement $B$ est cependant très attentif au thème du stage, au fait qu'il concerne les autres enseignants de l'établissement et veille à ce que les enseignants fassent profiter leurs collègues des bénéfices des séances de formation. Dans les deux autres Regelschulen, les chefs d'établissement ont organisé eux-mêmes l'année dernière des séances de formation dans l'établissement, en réponse aux attentes et aux problèmes des collègues.

Ces exemples montrent clairement que les chefs d'établissement sont en train de se créer des espaces de liberté. Ils ne sont encore que fort peu à même de les défendre. Le fait que les autorités de tutelle soient encore elles-mêmes dans les limbes de l'organisation leur facilite la tâche.

\section{La participation des parents et l'ouverture sur l'extérieur}

Les fêtes, les représentations théâtrales, les projets en commun et les «semaines projet $»^{5}$ sont des temps forts qui viennent compléter le cours afin que l'établissement soit plus qu'une simple entreprise de transmission du savoir et puisse devenir un lieu de vie pour l'ensemble de la communauté scolaire. Les trois chefs d'établissement se distinguent par ce souci d'organiser des manifestations "porteuses d'identité ». Ces manifestations doivent avoir un impact à l'extérieur et améliorer l'image de marque de 
l'établissement, ce qui est de plus en plus le cas, si l'on en croit les chefs d'établissement. d'exigences. Ils manquent eux-mêmes d'expérience en tant que chefs d'établissement et doivent participer à la construction et à la mise en place d'un nouveau système qui ne leur est pas familier. Ils doivent motiver leurs enseignants pour ce processus, alors que ces derniers ont, dans un premier temps, à se qualifier pour répondre aux nouvelles exigences, tant sur le plan des contenus que de la pédagogie. De plus, les conditions matérielles dans lesquelles ils doivent exercer sont souvent très difficiles. Le succès de leur entreprise dépend énormément des conditions matérielles et de la participation du corps professoral. Ce n'est donc pas un hasard si le chef d'établissement B, en cours d'entretien, a fait, en passant, la remarque suivante sur ses collègues: "C'est un heureux mélange!». Ce n'est qu'à cette condition que ce chef d'établissement a pu obtenir les résultats mentionnés dans un laps de temps aussi court, depuis la mutation complète du système éducatif de Thuringe.

Mais les deux autres chefs d'établissement fournissent aussi des efforts énormes pour faire avancer leurs établissements sur la voie de l'identité. Des influences extérieures, que ce soit une fusion d'établissements ou la menace d'une fermeture, ne manquent pas 
d'avoir des répercussions sur le soutien apporté par les enseignants et les parents. Ces exemples montrent particulièrement clairement la complexité et la durée du processus de mutation d'un système éducatif et la position difficile du chef d'établissement dans cette entreprise.

\section{NOTES}

1. La Gesamtschule (GS) est certes prévue, dans la loi secondaire de Thuringe, comme troisième type d'établissement de premier cycle du secondaire, mais ne doit venir qu'en complément des deux autres. Il y a actuellement trois GS en Thuringe.

2. Le système unique recevait l'approbation de la grande majorité des enseignants. Ils souhaitaient essentiellement des modifications de contenu (sans modification de structure) et plus de liberté pédagogique (cf. Hoffman/Chalupsky, 1991, Transformation des structures scolaires en Thuringe).

3. Au lieu de classes à profil, en fonction du diplôme envisagé, les établissements peuvent aussi (entre la septième et la neuvième classe) constituer des classes hétérogènes, avec des groupes de niveau dans les matières principales.

4. En Allemagne, le chef d'établissement a un rôle pédagogique, il assiste à certains cours et participe à l'évaluation des enseignants (NDT).

5. Ces "semaines projet», un classique de la pédagogie en Allemagne, sont organisées en collaboration avec les élèves. Il ne s'agit pas d'actions pédagogiques prévues seulement par les enseignants et la direction (NDT).

\section{RÉSUMÉS}

La réunification allemande a entraîné une restructuration complète des systèmes éducatifs dans les Länder de l'ancienne RDA. Les enjeux et les difficultés de ces transformations sont examinés à travers l'exemple de trois établissements d'un type nouveau - la Regelschule - dans le Land de Thuringe.

INDEX

Mots-clés : chef d'établissement, établissement d'enseignement, personnel d'encadrement Index géographique: Thuringe, Allemagne 


\section{AUTEURS}

\section{HORST WEISHAUPT}

Professeur en sciences de l'éducation, Pädagogische Hochschule, Erfurt, Allemagne

\section{MONIKA PLATH}

Pädagogische Hochschule, Erfurt, Allemagne

\section{ILONA BÖTTCHER}

Pädagogische Hochschule, Erfurt, Allemagne 